\title{
Structures of New Monoterpenes from Thai Herbal Medicine Curcuma comosa
}

\author{
Seikou Nakamura, ${ }^{a}$ Yang Qu, ${ }^{a, b}$ Fengming Xu,${ }^{a}$ Hisashi Matsuda,,${ }^{a}$ and Masayuki YoshiKawa ${ }^{*}, a$ \\ ${ }^{a}$ Kyoto Pharmaceutical University; Misasagi, Yamashina-ku, Kyoto 607-8412, Japan: and ${ }^{b}$ School of Traditional Chinese \\ Materia Medica, Shenyang Pharmaceutical University; Shenyang 110016, China. \\ Received June 26, 2008; accepted August 20, 2008; published online August 25, 2008
}

Three new monoterpenes, comosoxide A (1), comosoxide B (2), and comososide (3), were isolated from the methanolic extract of the rhizomes of Curcuma comosa cultivated in Thailand. Their structures were elucidated on the basis of chemical and physicochemical evidence.

Key words Curcuma comosa; Zingiberaceae; traditional Thai medicine; monoterpene; comosoxide; comososide

A Zingiberaceae plant, Curcuma comosa, is widely distributed in tropical and subtropical regions of the world, especially Thailand, Indonesia, and Malaysia. The rhizome of $C$. comosa has been used extensively as an indigenous medicine called "Waan chak mot luuk" in Thailand for the treatment of postpartum uterine bleeding. This medicine has also been used as an aromatic stomachic and anti-inflammatory agent. ${ }^{1,2)}$ In the course of our characterization studies on traditional Thai medicines, ${ }^{3-16)}$ we previously reported six new sesquiterpenes named $(+)$ - and $(-)$-comosols, comosones I, II, and III, and dimethoxycurcumenone from the methanolic extract of the rhizomes of $C$. comosa together with 26 known sesquiterpenes. ${ }^{17,18)}$ As a continuing study on this natural medicine, we additionally isolated three new monoterpenes, comosoxide A (1), comosoxide B (2), and comososide (3), together with 7 known compounds. This paper deals with the isolation and structure elucidation of three new constituents (1-3).

The methanolic extract from the dried rhizomes of $C$. $c o$ mosa (cultivated in Thailand) was partitioned into a mixture of ethyl acetate (EtOAc) and water to furnish the EtOAc-soluble fraction and an aqueous layer as previously described. ${ }^{18)}$ The EtOAc- and $n$-BuOH-soluble fractions were subjected to silica gel and octadecyl silica (ODS) column chromatography and finally HPLC to furnish three new monoterpenes, comosoxide A (1, 0.00055\%), comosoxide B (2, 0.00046\%), and comososide $(\mathbf{3}, 0.00028 \%)$, three known monoterpenes, 1-hydroxy- $\alpha, \alpha, 4$-trimethyl-3-cyclohexene-1-methanol ${ }^{19)}$ (4,

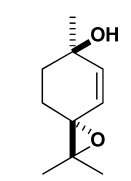

comosoxide A (1)
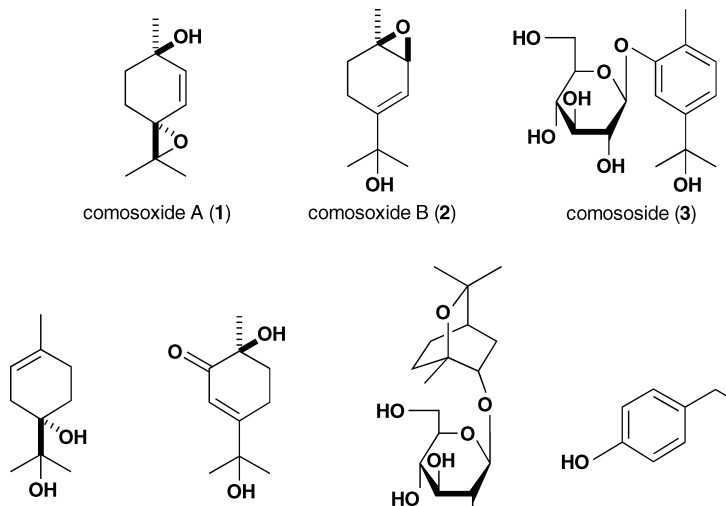

4
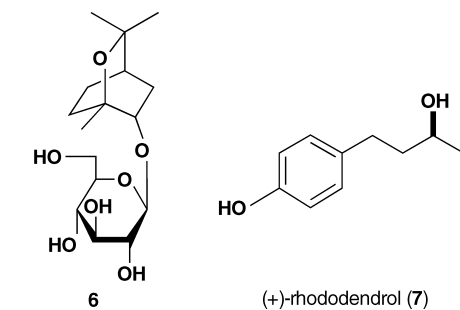

0.00021\%), 6-hydroxy-3-(1-hydroxyl-1-methylethyl)-6methyl-2-cyclohexen-1-one ${ }^{20)}(\mathbf{5}, 0.00037 \%),(1 S, 2 S, 4 R)-2-$ hydroxy-1,8-cineole $\beta$-D-glucopyranoside ${ }^{21)}(6,0.00020 \%)$, and four phenolic compounds, (+)-rhododendrol ${ }^{22)}$ (7, $0.0086 \%)$, 4-(4-hydroxyphenyl)butan-2-one ${ }^{23)}(0.00019 \%), 4-$ hydroxybenzaldehyde ${ }^{24)}(0.00049 \%)$, 4-hydroxy-3-methoxybenzaldehyde ${ }^{24)}(0.00022 \%)$.

Comosoxide A (1) was obtained as a colorless oil with positive optical rotation $\left([\alpha]_{\mathrm{D}}^{25}+16.0\right)$. In the electron impact (EI)-MS of 1, a molecular ion peak was observed at $\mathrm{m} / \mathrm{z} 168$ $\left(\mathrm{M}^{+}\right)$, and high-resolution EI-MS (HR-EI-MS) analysis revealed the molecular formula of $\mathbf{1}$ to be $\mathrm{C}_{10} \mathrm{H}_{16} \mathrm{O}_{2}$. Its IR spectrum showed absorption bands at 3400 and $1650 \mathrm{~cm}^{-1}$ ascribable to hydroxyl and olefin functions. The ${ }^{1} \mathrm{H}-$ and ${ }^{13} \mathrm{C}$ NMR $\left(\mathrm{CD}_{3} \mathrm{OD}\right.$, Table 1) spectra of $\mathbf{1}$ showed signals assignable to three tertiary methyls $[\delta 1.16,1.22,1.27$ (3H each, all s, 9, 10, 7- $\left.\mathrm{H}_{3}\right)$ ], two methylenes [ $\delta 1.56(1 \mathrm{H}$, ddd, $J=3.4,7.6$, $17.2 \mathrm{~Hz}, 5 \alpha-\mathrm{H}), 2.00(1 \mathrm{H}$, ddd, $J=2.8,13.1,17.2 \mathrm{~Hz}, 5 \beta-\mathrm{H})$, $1.68(1 \mathrm{H}$, ddd, $J=2.8,7.6,16.5 \mathrm{~Hz}, 6 \beta-\mathrm{H}), 1.88(1 \mathrm{H}, \mathrm{ddd}$, $J=3.4,13.1,16.5 \mathrm{~Hz}, 6 \alpha-\mathrm{H})]$, two methines $[\delta 5.73,5.89$ (1H each, both d, $J=10.3 \mathrm{~Hz}, 2,3-\mathrm{H})]$. The planar structure of 1 was confirmed by ${ }^{1} \mathrm{H}-{ }^{1} \mathrm{H}$ COSY and $\mathrm{HMBC}$ experiments. As shown in Fig. 1, the former indicated the presence of two partial structures written in the bold lines, while longrange correlations in the HMBC experiment on $\mathbf{1}$ were observed between the following proton and carbon pairs ( $\mathrm{H}-2$

Table 1. ${ }^{13} \mathrm{C}$-NMR Data for $\mathbf{1}-\mathbf{3}$

\begin{tabular}{|c|c|c|c|}
\hline & 1 & 2 & 3 \\
\hline C-1 & 67.4 & 72.5 & 126.9 \\
\hline C-2 & 136.9 & 74.8 & 154.6 \\
\hline C-3 & 131.1 & 122.3 & 113.0 \\
\hline C-4 & 74.4 & 147.1 & 149.9 \\
\hline C-5 & 27.9 & 24.2 & 119.4 \\
\hline C-6 & 34.4 & 34.7 & 131.2 \\
\hline C-7 & 30.3 & 21.9 & 16.1 \\
\hline C- 8 & 75.5 & 72.9 & 73.0 \\
\hline C-9 & 25.1 & 29.0 & 31.8 \\
\hline C-10 & 24.6 & 29.1 & 31.9 \\
\hline $\mathrm{C}-1^{\prime}$ & & & 102.8 \\
\hline C- $-2^{\prime}$ & & & 75.1 \\
\hline C-3' & & & 78.2 \\
\hline C- $-4^{\prime}$ & & & 71.5 \\
\hline C-5' & & & 78.2 \\
\hline C- $6^{\prime}$ & & & 62.7 \\
\hline
\end{tabular}

Measured in $\mathrm{CD}_{3} \mathrm{OD}$ at $150 \mathrm{MHz}$

Chart 1. Constituents Isolated from the Rhizomes of C. comosa 


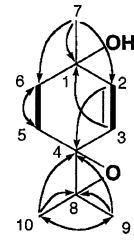

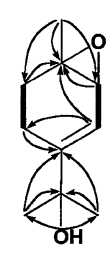

2

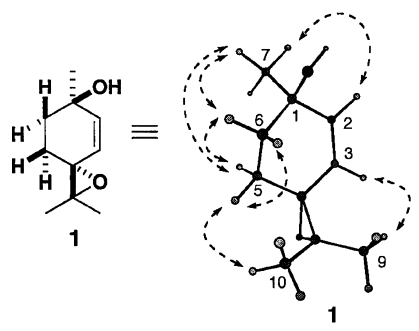

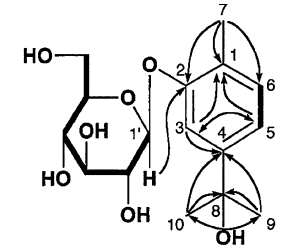

$3 \quad-{ }^{1} \mathrm{H}^{-1} \mathrm{H} \operatorname{COSY}$

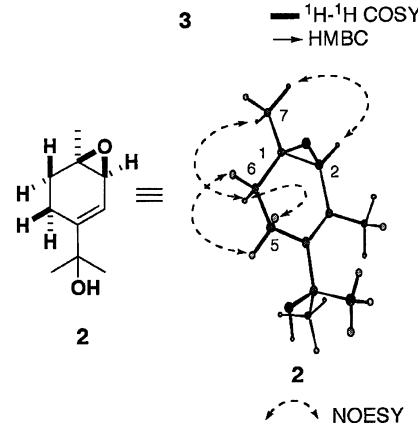

2
Fig. 1. Significant ${ }^{1} \mathrm{H}-{ }^{1} \mathrm{H}$ COSY, HMBC, and NOE Correlations for $\mathbf{1}-\mathbf{3}$

and $\mathrm{C}-4 ; \mathrm{H}-3$ and $\mathrm{C}-1 ; \mathrm{H}-5$ and $\mathrm{C}-6$; H-6 and $\mathrm{C}-5 ; \mathrm{H}_{3}-7$ and $\mathrm{C}-1,2,6 ; \mathrm{H}_{3}-9$ and $\mathrm{C}-4,8,10 ; \mathrm{H}_{3}-10$ and $\left.\mathrm{C}-4,8,9\right)$. The relative stereostructures of the 1 and 4-positions in $\mathbf{1}$ were clarified by a NOESY experiment, in which the NOE correlations were observed between the following proton pairs $(\mathrm{H}-2$ and $\mathrm{H}_{3}-7$; $\mathrm{H}-3$ and $\mathrm{H}_{3}-9$; $\mathrm{H}-5 \alpha$ and $\mathrm{H}-6 \alpha, \mathrm{H}_{3}-7$; $\mathrm{H}-5 \beta$ and $\mathrm{H}-6 \beta$, $\mathrm{H}_{3}-10 ; \mathrm{H}-6 \alpha$ and $\mathrm{H}_{3}-7$ ). Those findings led us to formulate the structure of comosoxide A (1) to be as shown. ${ }^{25}$ )

Comosoxide B (2) was isolated as a colorless oil with positive optical rotation $\left([\alpha]_{\mathrm{D}}^{27}+18.1\right)$. The EI-MS spectrum of 2 showed a molecular ion peak at $m / z 168\left(\mathrm{M}^{+}\right)$and the molecular formula of 2 was established as $\mathrm{C}_{10} \mathrm{H}_{16} \mathrm{O}_{2}$ by HR-EIMS measurement. Its IR spectrum suggested the presence of hydroxyl $\left(3370 \mathrm{~cm}^{-1}\right)$ and olefin $\left(1652 \mathrm{~cm}^{-1}\right)$ functions. The ${ }^{1} \mathrm{H}$ - and ${ }^{13} \mathrm{C}-\mathrm{NMR}\left(\mathrm{CD}_{3} \mathrm{OD}\right.$, Table 1$)$ spectra of $\mathbf{2}$ showed signals assignable to three tertiary methyls $[\delta 1.15,1.29,1.29$ $\left(3 \mathrm{H}\right.$ each, all s, $\left.\left.7,9,10-\mathrm{H}_{3}\right)\right]$, two methylenes $[\delta 1.64(1 \mathrm{H}$, ddd, $J=2.1,6.9,13.1 \mathrm{~Hz}, 6 \alpha-\mathrm{H}), 1.69(1 \mathrm{H}$, ddd, $J=5.5,11.7$, $13.1 \mathrm{~Hz}, 6 \beta-\mathrm{H}), 2.11(1 \mathrm{H}, \mathrm{m}, 5 \beta-\mathrm{H}), 2.24(1 \mathrm{H}, \mathrm{m}, 5 \alpha-\mathrm{H})]$, one methine bearing an oxygen function $[\delta 3.92(1 \mathrm{H}, \mathrm{d}$, $J=1.4 \mathrm{~Hz}, 2-\mathrm{H})]$, one olefin proton $[\delta 5.60(1 \mathrm{H}, \mathrm{d}, J=1.4 \mathrm{~Hz}$, $3-\mathrm{H})]$. The planar structure of 2 was constructed by ${ }^{1} \mathrm{H}-{ }^{1} \mathrm{H}$ COSY and HMBC experiments (Fig. 1). The relative configuration of $\mathbf{2}$ was determined by a NOESY experiment, in which the NOE correlations were observed between the following proton pairs ( $\mathrm{H}-2$ and $\mathrm{H}_{3}-7$; $\mathrm{H}-5 \alpha$ and $\mathrm{H}-6 \alpha$; $\mathrm{H}-5 \beta$ and $\mathrm{H}-6 \beta$; $\mathrm{H}-6 \alpha$ and $\left.\mathrm{H}_{3}-7\right)$. Thus, the structure of comosoxide B (2) was established to be as shown. ${ }^{25}$ )

Comososide (3) was isolated as a colorless powder with negative optical rotation $\left([\alpha]_{\mathrm{D}}^{26}-21.8\right)$. The molecular formula of 3 was established as $\mathrm{C}_{16} \mathrm{H}_{24} \mathrm{O}_{7}$ by HR-EI-MS measurement. Its IR spectrum showed absorption bands at 3365 , 1647 , and $1541 \mathrm{~cm}^{-1}$ ascribable to hydroxyl functions and aromatic ring. The UV spectrum of $\mathbf{3}$ showed absorption maxima at $210 \mathrm{~nm}(\log \varepsilon 4.54)$ and $277 \mathrm{~nm}$ (3.84), suggesting the presence of aromatic ring. The acid hydrolysis of 3 with $1 \mathrm{M} \mathrm{HCl}$ in 1,4-dioxane $(1: 1, \mathrm{v} / \mathrm{v})$ liberated D-glucose, which was identified by HPLC analysis using an optical rotation detector. ${ }^{16)}$ The ${ }^{1} \mathrm{H}$ - and ${ }^{13} \mathrm{C}$-NMR $\left(\mathrm{CD}_{3} \mathrm{OD}\right.$, Table 1$)$ spectra of 3 showed signals assignable to three methyls $[\delta 1.50,1.50$, $2.24\left(3 \mathrm{H}\right.$ each, all s, 9, 10, 7- $\left.\left.\mathrm{H}_{3}\right)\right]$, three ortho- and meta-coupled aromatic protons $[\delta 7.01(1 \mathrm{H}, \mathrm{dd}, J=7.8,1.8 \mathrm{~Hz}, 5-\mathrm{H})$, $7.25(1 \mathrm{H}, \mathrm{d}, J=1.8 \mathrm{~Hz}, 3-\mathrm{H}), 7.25(1 \mathrm{H}, \mathrm{d}, J=7.8 \mathrm{~Hz}, 6-\mathrm{H})]$ together with a glucopyranosyl moiety $\{\delta 4.89(1 \mathrm{H}, \mathrm{d}, J=7.7$ $\left.\mathrm{Hz}, 1^{\prime}-\mathrm{H}\right), 3.44,3.46,3.46,3.49$ (1H each, all m, 4', 3', 5', $\left.2^{\prime}-\mathrm{H}\right),[3.70(1 \mathrm{H}, \mathrm{dd}, J=12.0,5.5 \mathrm{~Hz}), 3.89(1 \mathrm{H}, \mathrm{dd}, J=12.0$, $\left.\left.1.7 \mathrm{~Hz}), 6^{\prime}-\mathrm{H}_{2}\right]\right\}$. The proton signals due to the aglycon part in the ${ }^{1} \mathrm{H}-\mathrm{NMR}$ data of $\mathbf{3}$ were superimposable on those of 3 hydroxy- $\alpha, \alpha, 4$-trimethyl benzyl alcohol. ${ }^{26}$ The position of the glycoside linkage was clarified by the HMBC experiment, which showed long-range correlation between the anomeric proton and the 2-carbon (Fig. 1). On the basis of these findings, the structure of $\mathbf{3}$ was determined to be as shown.

\section{Experimental}

The following instruments were used to obtain physical data: specific rotations, Horiba SEPA-300 digital polarimeter $(l=5 \mathrm{~cm})$; UV spectra, Shimadzu UV-1600 spectrometer; IR spectra, Shimadzu FTIR-8100 spectrometer; EI-MS and high-resolution EI-MS, JEOL JMS-GCMATE mass spectrometer; ${ }^{1} \mathrm{H}-\mathrm{NMR}$ spectra, JNM-LA500 $(500 \mathrm{MHz})$ and JNM-ECA600 $(600 \mathrm{MHz})$ spectrometers; ${ }^{13} \mathrm{C}-\mathrm{NMR}$ spectra, JNM-LA500 $(125 \mathrm{MHz})$ and JNM-ECL600 $(150 \mathrm{MHz})$ spectrometers with tetramethylsilane as an internal standard; and HPLC detector, Shimadzu RID-6A refractive index and SPD-10Avp UV-VIS detectors. HPLC column, COSMOSIL 5C18-PAQ $(250 \times 4.6 \mathrm{~mm}$ i.d. $)$ and $(250 \times 20 \mathrm{~mm}$ i.d. $)$ columns were used for analytical and preparative purposes, respectively.

The following experimental conditions were used for chromatography: ordinary-phase silica gel column chromatography, Silica gel BW-200 (Fuji Silysia Chemical, Ltd., Aichi, Japan, 150-350 mesh); reversed-phase silica gel column chromatography, Chromatorex ODS DM1020T (Fuji Silysia Chemical, Ltd., Aichi, Japan, 100-200 mesh); TLC, precoated TLC plates with Silica gel $60 \mathrm{~F}_{254}$ (Merck, $0.25 \mathrm{~mm}$ ) (ordinary phase) and Silica gel RP$18 \mathrm{~F}_{254 \mathrm{~S}}$ (Merck, $0.25 \mathrm{~mm}$ ) (reversed phase); reversed-phase HPTLC, precoated TLC plates with Silica gel RP-18 $\mathrm{WF}_{254 \mathrm{~S}}$ (Merck, $0.25 \mathrm{~mm}$ ); and detection was achieved by spraying with $1 \% \mathrm{Ce}\left(\mathrm{SO}_{4}\right)_{2}-10 \%$ aqueous $\mathrm{H}_{2} \mathrm{SO}_{4}$ followed by heating.

Plant Material Curcuma comosa was cultivated in Nakhon Si Thammarat of Thailand in July 2006, and identified by Dr. Yutana Pongpiriyadacha. A voucher specimen (No. T-32) is on file in our laboratory (Kyoto Pharmaceutical University, Department of Pharmacognosy).

Extraction and Isolation Fractions 5-2 (34 mg), 5-3 (79 mg), 5-6 (207 mg), 6 (26.99 g), 7-3 (78 mg), 8 (22.43 g), and 9 (4.59g) were obtained from the EtOAc-soluble fraction of the $\mathrm{MeOH}$ extract from the rhizomes of C. comosa $(4.5 \mathrm{~kg})$ as reported previously. ${ }^{18)}$ Fr. $5-2(34 \mathrm{mg})$ was purified by HPLC $\left[\mathrm{CH}_{3} \mathrm{OH}-\mathrm{H}_{2} \mathrm{O}(35: 65, \mathrm{v} / \mathrm{v})\right]$ to give 4-hydroxybenzaldehyde $(19.1 \mathrm{mg}, 0.00049 \%)$. Fr. $5-3(79 \mathrm{mg})$ was isolated by $\mathrm{HPLC}\left[\mathrm{CH}_{3} \mathrm{OH}-\right.$ $\left.\mathrm{H}_{2} \mathrm{O}(30: 70, \mathrm{v} / \mathrm{v})\right]$ to give 4-hydroxy-3-methoxybenzaldehyde $(8.6 \mathrm{mg}$, $0.00022 \%)$ and 4-(4-hydroxyphenyl)butan-2-one $(7.3 \mathrm{mg}, 0.00019 \%)$. Fr. 5 $6(207 \mathrm{mg})$ was further separated by HPLC $\left[\mathrm{CH}_{3} \mathrm{OH}-\mathrm{H}_{2} \mathrm{O}(40: 60, \mathrm{v} / \mathrm{v})\right]$ to give 1-hydroxy- $\alpha, \alpha, 4$-trimethyl-3-cyclohexene-1-methanol $(4,8.3 \mathrm{mg}$, $0.00021 \%)$. Fr. $6(26.99 \mathrm{~g})$ was subjected to reversed-phase silica gel column chromatography $\left[680 \mathrm{~g}, \quad \mathrm{MeOH}-\mathrm{H}_{2} \mathrm{O} \quad(25: 75 \rightarrow 30: 70 \rightarrow 35: 65 \rightarrow 40: 60\right.$, $\mathrm{v} / \mathrm{v}) \rightarrow \mathrm{MeOH})$ ] to give fifteen fractions [Fr. 6-1 (143 mg), Fr. 6-2 (2744 mg), Fr. 6-3 (561 mg), Fr. 6-4 (725 mg), Fr. 6-5 (819 mg), Fr. 6-6 (416 mg), Fr. 6-7 $(802 \mathrm{mg})$, Fr. 6-8 $(365 \mathrm{mg})$, Fr. 6-9 $(861 \mathrm{mg})$, Fr. 6-10 (3936 mg), Fr. 6-11 (1441 mg), Fr. 6-12 (317 mg), Fr. 6-13 (3938 mg), Fr. 6-14 (572 mg), and Fr. 6-15 $(4021 \mathrm{mg})]$. Fr. 6-4 $(50 \mathrm{mg})$ was purified by HPLC $\left[\mathrm{CH}_{3} \mathrm{OH}-\mathrm{H}_{2} \mathrm{O}\right.$ $(35: 65, \mathrm{v} / \mathrm{v})]$ to give $(+)$-rhododendrol $(7,23.3 \mathrm{mg}, 0.0086 \%)$. Fr. $7-3$ $(78 \mathrm{mg})$ was purified by HPLC $\left[\mathrm{CH}_{3} \mathrm{OH}-\mathrm{H}_{2} \mathrm{O}(15: 85, \mathrm{v} / \mathrm{v})\right]$ to give 6-hydroxy-3-(1-hydroxy-1-methylethyl)-6-methyl-2-cyclohexen-1-one $(5,14.4$ $\mathrm{mg}, 0.00037 \%)$. Fr. $8(22.43 \mathrm{~g})$ was subjected to reversed-phase silica gel column chromatography $\left[841 \mathrm{~g}, \mathrm{MeOH}-\mathrm{H}_{2} \mathrm{O}(20: 80 \rightarrow 25: 75 \rightarrow 30: 70 \rightarrow\right.$ $40: 60, \mathrm{v} / \mathrm{v}) \rightarrow \mathrm{MeOH})$ ] to give ten fractions [Fr. 8-1 (28 mg), Fr. 8-2 (108 mg), Fr. 8-3 (130 mg), Fr. 8-4 (374 mg), Fr. 8-5 (1246 mg), Fr. 8-6 (1003 mg), Fr. 8-7 (689 mg), Fr. 8-8 (1346 mg), Fr. 8-9 (1224 mg), and Fr. 8$10(12687 \mathrm{mg})]$. Fr. $8-2(54 \mathrm{mg})$ was purified by HPLC $\left[\mathrm{CH}_{3} \mathrm{OH}-\mathrm{H}_{2} \mathrm{O}\right.$ $(5: 95, \mathrm{v} / \mathrm{v})]$ to give comosoxide A $(1,10.8 \mathrm{mg}, 0.00055 \%)$. Fr. $9(4.59 \mathrm{~g})$ was subjected to reversed-phase silica gel column chromatography $[150 \mathrm{~g}$, $\left.\left.\mathrm{MeOH}-\mathrm{H}_{2} \mathrm{O}(20: 80 \rightarrow 25: 75 \rightarrow 30: 70 \rightarrow 35: 65, \mathrm{v} / \mathrm{v}) \rightarrow \mathrm{MeOH}\right)\right]$ to give five fractions [Fr. 9-1 (130 mg), Fr. 9-2 (48 mg), Fr. 9-3 (660 mg), Fr. 9-4 
(542 mg), and Fr. 9-5 (2294 mg)]. Fr. 9-1 (130 mg) was purified by HPLC $\left[\mathrm{CH}_{3} \mathrm{OH}-\mathrm{H}_{2} \mathrm{O}(15: 85, \mathrm{v} / \mathrm{v})\right]$ to give comosoxide $\mathrm{B}(2,18.0 \mathrm{mg}, 0.00046 \%)$. Fr. 9-2 $(48 \mathrm{mg})$ was isolated on HPLC to give $(1 S, 2 S, 4 R)$-2-hydroxy-1,8-cineole $\beta$-D-glucopyranoside $(6,7.8 \mathrm{mg}, 0.00020 \%)$.

The MeOH-eluted fraction $(7.0 \mathrm{~g})$ was subjected to reversed-phase silica gel column chromatography $\left[210 \mathrm{~g}, \mathrm{MeOH}-\mathrm{H}_{2} \mathrm{O}(5: 95 \rightarrow 10: 90 \rightarrow 15: 85 \rightarrow\right.$ $20: 80, \mathrm{v} / \mathrm{v}) \rightarrow \mathrm{MeOH})$ ] to give seven fractions [Fr. 12-1 (561 mg), Fr. 12-2 $(66 \mathrm{mg})$, Fr. $12-3(136 \mathrm{mg})$, Fr. $12-4(136 \mathrm{mg})$, Fr. $12-5$ (238 mg), Fr. $12-6$ $(200 \mathrm{mg})$, and Fr. 12-7 (405 mg)]. Fr. 12-7 (405 mg) was further isolated by $\mathrm{HPLC}\left[\mathrm{CH}_{3} \mathrm{OH}-\mathrm{H}_{2} \mathrm{O}(20: 80, \mathrm{v} / \mathrm{v})\right]$ to give comososide $(3,11.5 \mathrm{mg}$, $0.00028 \%$ ).

The known compounds [4-7, 4-(4-hydroxyphenyl)butan-2-one, 4-hydroxybenzaldehyde, and 4-hydroxy-3-methoxybenzaldehyde] were identified by comparison of their physical data $\left([\alpha]_{\mathrm{D}}\right.$, IR, $\left.{ }^{1} \mathrm{H}-\mathrm{NMR},{ }^{13} \mathrm{C}-\mathrm{NMR}, \mathrm{MS}\right)$ with reported values ${ }^{19-23)}$ or those of commercial samples. ${ }^{24)}$

Comosoxide A (1): Colorless oil, $[\alpha]_{\mathrm{D}}^{25}+16.0(c=0.6, \mathrm{MeOH})$. HR-EIMS: Calcd for $\mathrm{C}_{10} \mathrm{H}_{16} \mathrm{O}_{2}\left(\mathrm{M}^{+}\right)$168.1150; Found 168.1157. IR (film, $\mathrm{cm}^{-1}$ ): $3468,1458,1030 .{ }^{1} \mathrm{H}-\mathrm{NMR}\left(600 \mathrm{MHz}, \mathrm{CD}_{3} \mathrm{OD}\right) \delta: 1.16,1.22,1.27(3 \mathrm{H}$ each, all s, 9, 10, 7- $\left.\mathrm{H}_{3}\right), 1.56(1 \mathrm{H}$, ddd, $J=3.4,7.6,17.2 \mathrm{~Hz}, 5 \alpha-\mathrm{H}), 2.00$ $(1 \mathrm{H}, \mathrm{ddd}, J=2.8,13.1,17.2 \mathrm{~Hz}, 5 \beta-\mathrm{H}), 1.68(1 \mathrm{H}, \mathrm{ddd}, J=2.8,7.6,16.5 \mathrm{~Hz}$, $6 \beta-\mathrm{H}), 1.88(1 \mathrm{H}$, ddd, $J=3.4,13.1,16.5 \mathrm{~Hz}, 6 \alpha-\mathrm{H}), 5.73,5.89(1 \mathrm{H}$ each, both d, $J=10.3 \mathrm{~Hz}, 2,3-\mathrm{H}) .{ }^{13} \mathrm{C}-\mathrm{NMR}\left(150 \mathrm{MHz}, \mathrm{CD}_{3} \mathrm{OD}\right) \delta_{\mathrm{C}}$ : given in Table 1. EI-MS $(m / z, \%): 168\left(\mathrm{M}^{+}, 3\right), 110(100)$

Comosoxide B (2): Colorless oil, $[\alpha]_{\mathrm{D}}^{27}+18.1(c=1.1, \mathrm{MeOH})$. HR-EIMS: Calcd for $\mathrm{C}_{10} \mathrm{H}_{16} \mathrm{O}_{2}\left(\mathrm{M}^{+}\right)$168.1150; Found 168.1154. IR (film, $\mathrm{cm}^{-1}$ ): $3370,1458,1026 .{ }^{1} \mathrm{H}-\mathrm{NMR}\left(600 \mathrm{MHz}, \mathrm{CD}_{3} \mathrm{OD}\right) \delta: 1.15,1.29,1.29(3 \mathrm{H}$ each, all s, 7, 9, 10- $\left.\mathrm{H}_{3}\right), 1.64(1 \mathrm{H}$, ddd, $J=2.1,6.9,13.1 \mathrm{~Hz}, 6 \alpha-\mathrm{H}), 1.69$ $(1 \mathrm{H}$, ddd, $J=5.5,11.7,13.1 \mathrm{~Hz}, 6 \beta-\mathrm{H}), 2.11(1 \mathrm{H}, \mathrm{m}, 5 \beta-\mathrm{H}), 2.24(1 \mathrm{H}, \mathrm{m}$, $5 \alpha-\mathrm{H}), 3.92(1 \mathrm{H}, \mathrm{d}, J=1.4 \mathrm{~Hz}, 2-\mathrm{H}), 5.60(1 \mathrm{H}, \mathrm{d}, J=1.4 \mathrm{~Hz}, 3-\mathrm{H}) .{ }^{13} \mathrm{C}-\mathrm{NMR}$ $\left(150 \mathrm{MHz}, \mathrm{CD}_{3} \mathrm{OD}\right) \delta_{\mathrm{C}}$ : given in Table 1. EI-MS $(\mathrm{m} / \mathrm{z}, \%): 168\left(\mathrm{M}^{+}, 8\right), 95$ (100)

Comososide (3): Colorless powder, $[\alpha]_{\mathrm{D}}^{26}-21.8(c=0.8, \mathrm{MeOH})$. HR-EIMS: Calcd for $\mathrm{C}_{16} \mathrm{H}_{24} \mathrm{O}_{7}\left(\mathrm{M}^{+}\right)$328.1522; Found 328.1521. UV [MeOH, nm, $(\log \varepsilon)$ ]: 210 (4.54), 277 (3.84). IR (film, $\mathrm{cm}^{-1}$ ): 3365, 2932, 1541, 1246, $1035,771 .{ }^{1} \mathrm{H}-\mathrm{NMR}\left(600 \mathrm{MHz}, \mathrm{CD}_{3} \mathrm{OD}\right) \delta: 1.50,1.50,2.24(3 \mathrm{H}$ each, all s, 9, 10, 7- $\left.\mathrm{H}_{3}\right), 3.44,3.46,3.46,3.49$ (1H each, all m, 4', 3', 5', $\left.2^{\prime}-\mathrm{H}\right),[3.70$ $\left.(1 \mathrm{H}, \mathrm{dd}, J=12.0,5.5 \mathrm{~Hz}), 3.89(1 \mathrm{H}, \mathrm{dd}, J=12.0,1.7 \mathrm{~Hz}), 6^{\prime}-\mathrm{H}_{2}\right], 4.89(1 \mathrm{H}, \mathrm{d}$, $\left.J=7.7 \mathrm{~Hz}, 1^{\prime}-\mathrm{H}\right), 7.01(1 \mathrm{H}, \mathrm{dd}, J=7.8,1.8 \mathrm{~Hz}, 5-\mathrm{H}), 7.25(1 \mathrm{H}, \mathrm{d}, J=1.8 \mathrm{~Hz}$, $3-\mathrm{H}), 7.25(1 \mathrm{H}, \mathrm{d}, J=7.8 \mathrm{~Hz}, 6-\mathrm{H}) .{ }^{13} \mathrm{C}-\mathrm{NMR}\left(150 \mathrm{MHz}, \mathrm{CD}_{3} \mathrm{OD}\right) \delta_{\mathrm{C}}$ : given in Table 1. EI-MS ( $m / z, \%): 328\left(\mathbf{M}^{+}, 1\right), 148$ (100).

Acid Hydrolysis of Comososide (3) A solution of $3(2.0 \mathrm{mg})$ in $1 \mathrm{~m}$ $\mathrm{HCl}$ in 1,4-dioxane $(1: 1, \mathrm{v} / \mathrm{v}, 0.5 \mathrm{ml})$ was heated under reflux for $2 \mathrm{~h}$. After cooling, the reaction mixture was poured into ice-water and neutralized with Amberlite IRA-400 $\left(\mathrm{OH}^{-}\right.$form $)$, and the resin was removed by filtration. Then, the filtrate was extracted with EtOAc. The aqueous layer was subjected to HPLC analysis under the following conditions: HPLC column, Kaseisorb $\mathrm{LC} \mathrm{NH}_{2}-60-5,4.6 \mathrm{~mm}$ i.d. $\times 250 \mathrm{~mm}$ (Tokyo Kasei Co., Ltd., Tokyo, Japan); detection, optical rotation [Shodex OR-2 (Showa Denko Co., Ltd., Tokyo, Japan)]; mobile phase, $\mathrm{CH}_{3} \mathrm{CN}-\mathrm{H}_{2} \mathrm{O}(85: 15$, v/v); flow rate $0.8 \mathrm{ml} / \mathrm{min}$; column temperature, room temperature. Identification of D-glucose from 3 present in the aqueous layer was carried out by comparison of its retention time and optical rotation with those of authentic sample. $t_{\mathrm{R}}$ : $12.7 \mathrm{~min}$ (positive optical rotation).

Acknowledgments This research was supported by the 21st COE Program, Academic Frontier Project, and a Grant-in Aid for Scientific Research from MEXT (the Ministry of Education, Culture, Sports, Science and Technology of Japan).

\section{References and Notes}

1) Sodsai A., Piyachaturawat P., Sophasan S., Suksamrarn A., Vongsakul M., Int. Immunopharmacol., 7, 524-531 (2007).

2) Jantaratnotai N., Utaisincharoen P., Piyachaturawat P., Chongthammakun S., Sanvarinda Y., Life Sci., 78, 571-577 (2006).

3) Yoshikawa M., Morikawa T., Nakano K., Pongpiriyadacha Y., Murakami T., Matsuda H., J. Nat. Prod., 65, 1638-1642 (2002).

4) Matsuda H., Pongpiriyadacha Y., Morikawa T., Ochi M., Yoshikawa M., Eur. J. Pharmacol., 471, 59-67 (2003).

5) Matsuda H., Morikawa T., Managi H., Yoshikawa M., Bioorg. Med. Chem. Lett., 13, 3197-3202 (2003).

6) Morikawa T., Kishi A., Pongpiriyadacha Y., Matsuda H., Yoshikawa M., J. Nat. Prod., 66, 1191-1196 (2003).

7) Kishi A., Morikawa T., Matsuda H., Yoshikawa M., Chem. Pharm. Bull., 51, 1051-1055 (2003).

8) Yoshikawa M., Pongpiriyadacha Y., Kishi A., Kageura T., Wang T., Morikawa T., Matsuda H., Yakugaku Zasshi, 123, 871-880 (2003).

9) Matsuda H., Tewtrakul S., Morikawa T., Nakamura A., Yoshikawa M., Bioorg. Med. Chem., 12, 5891-5898 (2004).

10) Morikawa T., Matsuda H., Yamaguchi I., Pongpiriyadacha Y., Yoshikawa M., Planta Med., 70, 152-159 (2004).

11) Matsuda H., Morikawa T., Xu F., Ninomiya K., Yoshikawa M., Planta Med., 70, 1201-1209 (2004).

12) Matsuda H., Ando S., Morikawa T., Kataoka S., Yoshikawa M., Bioorg. Med. Chem. Lett., 15, 1949-1953 (2005).

13) Ando S., Matsuda H., Morikawa T., Yoshikawa M., Bioorg. Med. Chem., 13, 3289-3294 (2005).

14) Morikawa T., Xu F., Matsuda H., Yoshikawa M., Chem. Pharm. Bull., 54, 1530-1534 (2006)

15) Matsuda H., Yoshida K., Miyagawa K., Asao Y., Takayama S., Nakashima S., Xu F., Yoshikawa M., Bioorg. Med. Chem., 15, 15391546 (2007).

16) Yoshikawa M., Xu F., Morikawa T., Pongpiriyadacha Y., Nakamura S., Asao Y., Kumahara A., Matsuda H., Chem. Pharm. Bull., 55, 308316 (2007).

17) Qu Y., Xu F., Nakamura S., Matsuda H., Pongpiriyadacha Y., Wu L., Yoshikawa M., J. Nat. Med., accepted.

18) Xu F., Nakamura S., Qu Y., Matsuda H., Pongpiriyadacha Y., Wu L., Yoshikawa M., Chem. Pharm. Bull., submitted.

19) Carman R. M., Rayner A. C., Aust. J. Chem., 47, 2087-2097 (1994).

20) Asakawa Y., Takahashi H., Toyota M., Noma Y., Phytochemistry, 30, 3981-3987 (1991)

21) Ishikawa T., Kitajima J., Tanaka Y., Ono M., Ito Y., Nohara T., Chem. Pharm. Bull., 46, 1738-1742 (1998).

22) Rojatkar S. R., Sawaikar D. D., Sinha B., Ravindranathan T., Nagasampagi B. A., Phytochemistry, 39, 259-260 (1995).

23) Marco J. A., Barbera O., Rodriguze S., Domingo C., Adell J., Phytochemistry, 27, 3155-3159 (1988).

24) 4-Hydroxybenzaldehyde and vanillin were identified by comparison of their physical data with those of commercial samples.

25) The absolute configurations of $\mathbf{1}$ and $\mathbf{2}$ have not been characterized yet.

26) Patwardhan S. A., Gupta A. S., Phytochemistry, 22, 2080-2081 (1983). 